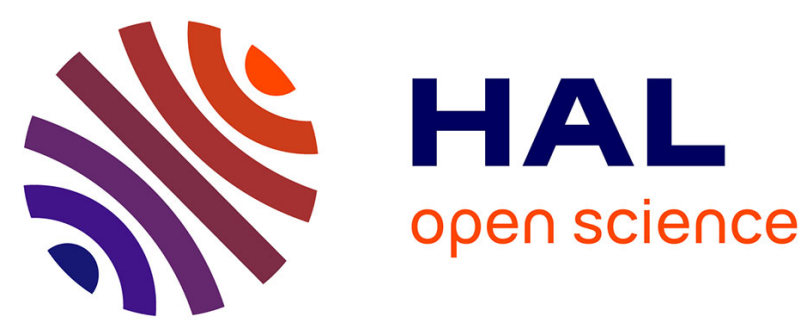

\title{
Recent measurements of the spherical and deformed isomers using the Lohengrin fission-fragment spectrometer
}

G.S. Simpson, J.C. Angélique, J. Genevey, J.A. Pinston, A. Covello, A. Gargano, U. Köster, R. Orlandi, A. Scherillo, W. Urban

\section{To cite this version:}

G.S. Simpson, J.C. Angélique, J. Genevey, J.A. Pinston, A. Covello, et al.. Recent measurements of the spherical and deformed isomers using the Lohengrin fission-fragment spectrometer. Fourth International Conference Fission and properties of neutron-rich nuclei, Nov 2007, Sanibel Island, United States. pp.71-79. in2p3-00376716

\section{HAL Id: in2p3-00376716 https://hal.in2p3.fr/in2p3-00376716}

Submitted on 20 Apr 2009

HAL is a multi-disciplinary open access archive for the deposit and dissemination of scientific research documents, whether they are published or not. The documents may come from teaching and research institutions in France or abroad, or from public or private research centers.
L'archive ouverte pluridisciplinaire HAL, est destinée au dépôt et à la diffusion de documents scientifiques de niveau recherche, publiés ou non, émanant des établissements d'enseignement et de recherche français ou étrangers, des laboratoires publics ou privés. 


\title{
RECENT MEASUREMENTS OF SPHERICAL AND DEFORMED ISOMERS USING THE LOHENGRIN FISSION-FRAGEMENT SPECTROMETER
}

\author{
G. S. SIMPSON, J. C. ANGELIQUE, J. GENEVEY and J. A. PINSTON
}

LPSC, Université Joseph Fourier Grenoble 1, CNRS/IN2P3, Institut National Polytechnique de Grenoble, F-38026 Grenoble Cedex, France

\section{A. COVELLO and A. GARGANO}

Dipartimento di Scienze Fisiche, Università degli Studi di Napoli Federico II and INFN, Complesso Universitario di Monte Sant'Angelo, Via Cintia, I-80126 Napoli, Italy

\section{U. KÖSTER}

Institut Laue-Langevin, B.P. 156, F-38042 Grenoble Cedex 9, France

$$
\text { R. ORLANDI }
$$

INFN, Laboratori Nazionali di Legnaro, Legnaro, Italy

$$
\text { A. SCHERILLO }
$$

Rutherford Appleton Laboratory, Chilton, Didcot OX11 oQX, United Kingdom

$$
\text { W. URBAN }
$$

Faculty of Physics, Warsaw University, ul.Hoża 69, PL-00-681 Warszawa, Poland

New experimental information has been obtained on the $\mu$ s isomeric cascade in the very neutron-rich ${ }^{136} \mathrm{Sb}$ using $\gamma$-ray and conversion-electron spectroscopy at the Lohengrin fission-product spectrometer of the Institut LaueLangevin, Grenoble. Two new transitions have been observed and their multipolarities determined, resolving the question of the origin of the isomerism. The new level scheme is in good agreement with predictions of a realistic shell-model calculation. Microsecond isomers in deformed neutron-rich fission fragments have also been studied in ${ }^{107} \mathrm{Mo}$ and ${ }^{107} \mathrm{Tc}$ with the Lohengrin spectrometer. These studies have been complemented by prompt $\gamma$-ray spectroscopy of these nuclei, plus the neighboring ${ }^{105} \mathrm{Mo}$, following the spontaneous fission of a ${ }^{248} \mathrm{Cm}$ source inside EUROGAM2. Simple quasiparticle-rotor model calculations are able to reproduce the experimental level schemes and decay patterns. 


\section{1. ${ }^{136} \mathrm{Sb}$}

\subsection{Introduction}

Recent shell-model studies employing a two-body effective interaction derived from the CD-Bonn nucleon-nucleon $(N N)$ potential, with no use of any adjustable parameter have been able to successfully reproduce the experimental level schemes of ${ }^{134,135} \mathrm{Sb}[1,2]$. The $\mathrm{Sb}$ isotopes are most appropriate for testing the matrix elements of the proton-neutron interaction between valence nucleons in different major shells. These calculations show that there is no need to invoke shell-structure modifications to explain the presently available data on neutron-rich nuclei beyond ${ }^{132} \mathrm{Sn}$. The nucleus ${ }^{136} \mathrm{Sb}$, with an $\mathrm{N} / \mathrm{Z}$ ratio of 1.67 , is at present the most exotic open-shell nucleus beyond ${ }^{132} \mathrm{Sn}$ for which a spectroscopic study has been performed [3]. In this study it was concluded that the $\mu$ s isomer observed most likely originates from the $I^{\pi}=6^{-}$state of the $\pi g_{7 / 2} \nu\left(f_{7 / 2}\right)^{3}$ configuration. Only one $\gamma$-ray transition of $173 \mathrm{keV}$ was observed in coincidence with ${ }^{136} \mathrm{Sb}$ ions, which alone was not enough to explain the origin of $\mu$ s isomerism. In Ref. [3] an unseen low-energy $E 2$ transition was postulated to be responsible for the $\mu$ s lifetime. In the present work this nucleus has been studied with an experimental setup capable of detecting low-energy conversion electrons and $\gamma$ rays.

\subsection{Experimental Method}

Delayed $\gamma$ rays and conversion electrons from ${ }^{136} \mathrm{Sb}$ were observed using the Lohengrin mass spectrometer at the high-flux reactor of the Institut LaueLangevin, Grenoble. Mass 136 nuclei were produced by thermal-neutron induced fission of a thin $7 \times 0.5 \mathrm{~cm}^{2}, 1 \mathrm{mg}{ }^{241} \mathrm{Pu}$ target. The Lohengrin mass spectrometer was used to select nuclei recoiling from the target, according to their mass-to-ionic charge ratios $(A / q)$. The flight time of the $A=136$ nuclei through the spectrometer was around $2.3 \mu \mathrm{s}$. The energy of the fission fragments was detected in a $\Delta E 1 \Delta E 2$ ionization chamber, filled with isobutane gas, allowing the identification of $A / q$. The pressure of the gas in the chamber was adjusted so that the fission products stopped in the last few $\mu \mathrm{m}$ of a $6 \mu \mathrm{m}$ thick Mylar foil. A few $\mathrm{mm}$ behind the foil, two adjacent, rectangular, liquid-nitrogen cooled $\mathrm{Si}(\mathrm{Li})$ detectors were placed, to detect $\mathrm{X}$ rays and conversion electrons down to about $20 \mathrm{keV}$ in energy. $\gamma$ rays de-exciting isomeric states, and states below the isomer, were detected by two Clover Ge detectors. The total $\gamma$-ray detection efficiency was $14.6 \%$ and $3.5 \%$ for photons of $100 \mathrm{keV}$ and $1 \mathrm{MeV}$, respectively. Any 
$\gamma$ rays detected in the Ge or Si detectors up to $20 \mu$ s after the arrival of an ion were recorded by the the data acquisition system. Measurements of delayed $\gamma$ rays and conversion electrons from ${ }^{136} \mathrm{Sb}$ were an experimental challenge as the ionization chamber was unable to resolve the different isobars in the $A=136$ mass chain. Another $\mu$ s isomer exists in this mass chain, the $2.95 \mu \mathrm{s} 6^{+}$state ${ }^{136} \mathrm{Xe}$ [4], whose fission yield is a factor 25 higher than that of ${ }^{136} \mathrm{Sb}$. The previously measured short lifetime of the isomer $(565(50) \mathrm{ns})$ [3], meant also that much of the intensity of the isomeric state decayed during the $2.3 \mu$ s flight time through the spectrometer. Hence, delayed $\gamma$-ray and conversion-electron spectra were dominated by transitions from the ${ }^{136} \mathrm{Xe}$ isomer.

\subsection{Results}

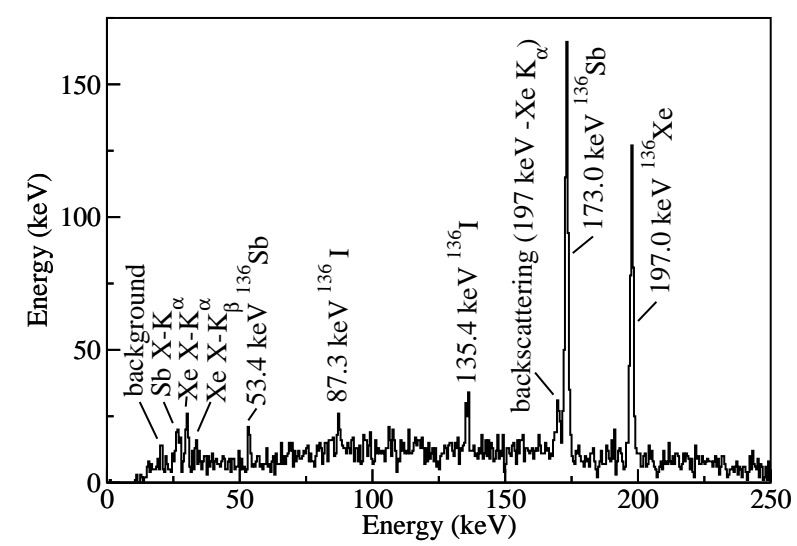

Fig. 1. $\gamma$ rays observed in the Ge detectors in coincidence with $\mathrm{Sb} K_{\alpha} \mathrm{X}$ rays measured in the Si detectors. Background transitions are present from the strongly produced isomer in ${ }^{136} \mathrm{Xe}[4]$ and from ${ }^{136} \mathrm{I}$, produced by $\beta$ decay

In addition to the previously reported delayed $173 \mathrm{keV} \gamma$ ray from ${ }^{136} \mathrm{Sb}$ [3], two new delayed transitions of 53.4(3) keV and 51.4(5) keV have been observed. The $53.4 \mathrm{keV} \gamma$ ray was observed in coincidence with $\mathrm{Sb} K_{\alpha} \mathrm{X}$ rays and $A=136$ ions, as shown in Fig. 1 . By gating on the $173.0 \mathrm{keV}$ $\gamma$-ray transition, observed in the Ge detectors, and $A=136$ ions it was possible to observe coincident conversion electrons at $47.1 \mathrm{keV}$ (corrected for the energy loss in the Mylar foil), as shown in Fig. 2. These electrons correspond to the $L+M$ groups, of the two new transitions. Correcting for 
the weighted mean $L$ binding energy for Sb and the energy loss in the Mylar foil gives a transition energy of $51.4(5) \mathrm{keV}$. By examining the $\gamma$ ray and conversion-electron intensity ratios it was possible to assign M1 and E2 multipolarities to the 53.4 and $51.4 \mathrm{keV}$ transitions respectively. More details on the assignments of these multipolarities are given in Ref. [5]. The halflife of the isomeric state was measured to be $480(100)$ ns, as shown in Fig. 3, in agreement with 565(50)ns measured in Ref. [3].

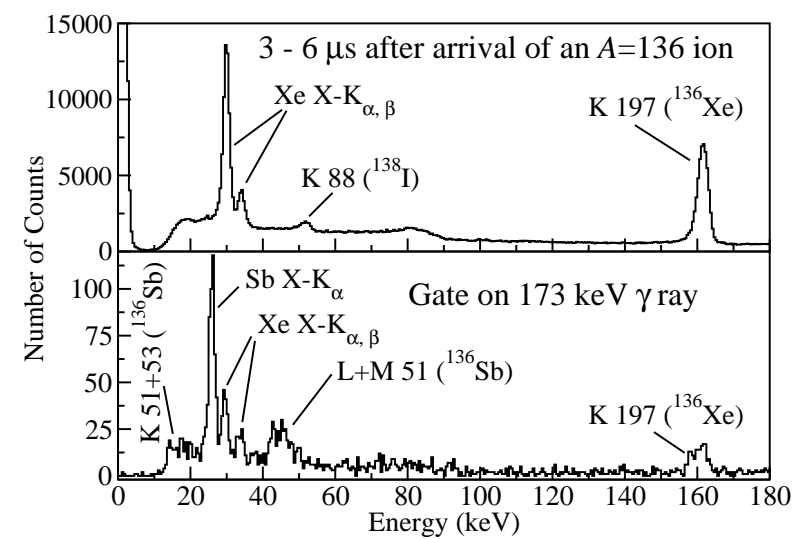

Fig. 2. Conversion electrons and X-rays observed in the Si detectors, in the upper spectrum 3 to $6 \mu$ s after the arrival of an $A=136$ ion and in the lower spectrum in coincidence with the $173.0 \mathrm{keV} \gamma$ ray in the Ge detectors, 0 to $1.5 \mu$ s after the arrival of an $A=136$ ion. A conversion-electron line of $47.1 \mathrm{keV}$ is observed and is identified as the $L+M$ lines of a $51.4 \mathrm{keV}$ transition from ${ }^{136} \mathrm{Sb}$.

\subsection{Discussion}

A shell-model calculation has been performed with the same realistic interaction used for ${ }^{134} \mathrm{Sb}$ and ${ }^{135} \mathrm{Sb}[1,2]$ and is described in the proceedings of A. Covello at this conference and in [5]. The proposed experimental level scheme of ${ }^{136} \mathrm{Sb}$ is shown in Fig. 4, where it is compared with the calculated results. In this figure the first four calculated states are reported and each of them has a counterpart in the experimental spectrum. The $51.4 \mathrm{keV} \mathrm{E2}$ is almost certainly the $6^{-} \rightarrow 4^{-}$isomeric transition, from lifetime arguements. The order of the $173 \mathrm{keV}$ and $51.4 \mathrm{keV}$ transitions has been assigned by comparison with the calculation. Note that the ground state spin was already identified as $1^{-}$in Ref. [6]. 


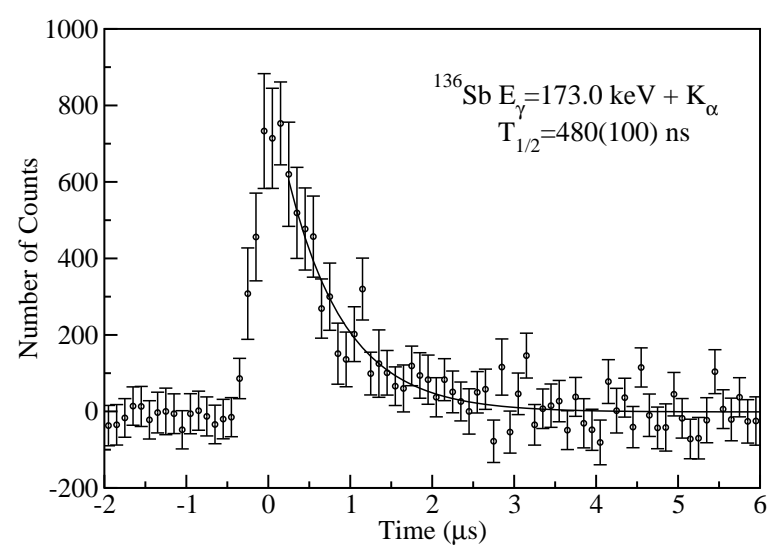

Fig. 3. Summed time spectrum of gates on the $173.0 \mathrm{keV} \gamma$-ray transition detected in the Clover Ge detectors and the $\mathrm{Sb} K_{\alpha}$ X-rays in the Si detectors.

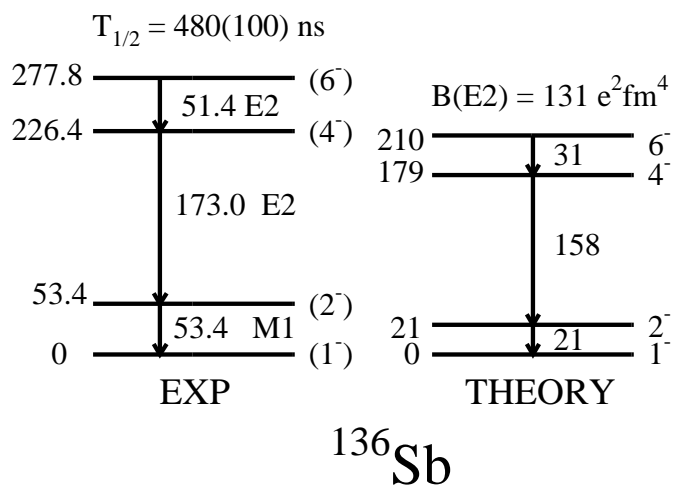

Fig. 4. Proposed decay scheme of the ${ }^{136} \mathrm{Sb}$ isomer.

The four observed levels in ${ }^{136} \mathrm{Sb}$ are all identified with states which are dominated by the configuration $\pi g_{7 / 2} \nu\left(f_{7 / 2}\right)^{3}$. It is worth mentioning that the percentages of configurations other than the dominant one in the considered states of ${ }^{136} \mathrm{Sb}$ are rather large, ranging from $24 \%$ to $32 \%$.

An important piece of information is provided by the measured halflife of the $6^{-}$state, from which a $B\left(E 2 ; 6^{-} \rightarrow 4^{-}\right)$value of $170(40) e^{2} \mathrm{fm}^{4}=4.2$ W.u. is extracted. Using effective proton and neutron charges of $1.55 e$ and $0.70 e$, respectively, we obtain the value $131 e^{2} \mathrm{fm}^{4}$, which compares very well 
with experiment. It is worth mentioning that these values of the effective charges have been consistently used in previous calculations for nuclei in the ${ }^{132} \mathrm{Sn}$ region [7].

\subsection{Summary}

Delayed $\gamma$ rays and conversion electrons have been measured in the very neutron-rich nucleus ${ }^{136} \mathrm{Sb}$, which represents a further step in the far-fromstability region beyond $\mathrm{N}=82$. The multipolarities of these transitions have been determined and a level scheme constructed by comparison with the results of a realistic shell-model calculation. The predicted energies and $B\left(E 2 ; 6^{-} \rightarrow 4^{-}\right)$are in very good agreement with measured values. This shows that a consistent shell-model description can be given of the presently known nuclei in this region.

\section{2. ${ }^{105,107} \mathrm{Mo},{ }^{107} \mathrm{Tc}$}

\subsection{Introduction}

The rapid shape change of the low lying states in the $\mathrm{Sr}$ and $\mathrm{Zr}$ nuclei, from spherical to deformed, when the neutron number increases from 58 to 60 neutrons is well known. In the nearby even-even ${ }^{104-108}$ Mo a new situation occurs. These nuclei are strongly deformed [8-10] but, at the same time, the levels of the $K^{\pi}=2^{+}, \gamma$-band descrease in energy with increasing neutron number, suggesting that the triaxial degree of freedom plays an important role in these isotopes. However, the nature of this triaxiality is not well understood. Calculations by Skalski et al. [11] predict ground state triaxial minima, with $\gamma=19-21^{\circ}$ for these nuclei. In constrast Smith et al. suggest that the triaxiality is dynamic in nature [8], with $\gamma \sim 0^{\circ}$ at low spins and a rotation-induced change occurring around $J \sim 10-12 \hbar$, the latter being supported by the experimental observation of a decrease in the quadrupole moment. To better understand the nature of this non-axial deformation it is also important to study the structure of the odd Mo nuclei. The nuclei ${ }^{105} \mathrm{Mo}$ and ${ }^{107}$ Mo have been revisited experimentally in the present work and the structure of these nuclei was analyzed in the framework of the particle-rotor coupling model. Similarly the influence of an odd-proton on the even-even core was examined by studying ${ }^{107} \mathrm{Tc}$.

\subsection{Experimental Method and Results}

The nuclei ${ }^{105,107} \mathrm{Mo}$ and ${ }^{107} \mathrm{Tc}$ were studied by $\gamma$-ray spectroscopy following the spontaneous fission of ${ }^{248} \mathrm{Cm}$ using the EUROGAM2 array. Previ- 
ously, four different configurations were proposed in ${ }^{105}$ Mo however only one well-developed band was observed which fed the ground state [10]. In the present work four well developed bands were observed in ${ }^{105} \mathrm{Mo}$.

(2)

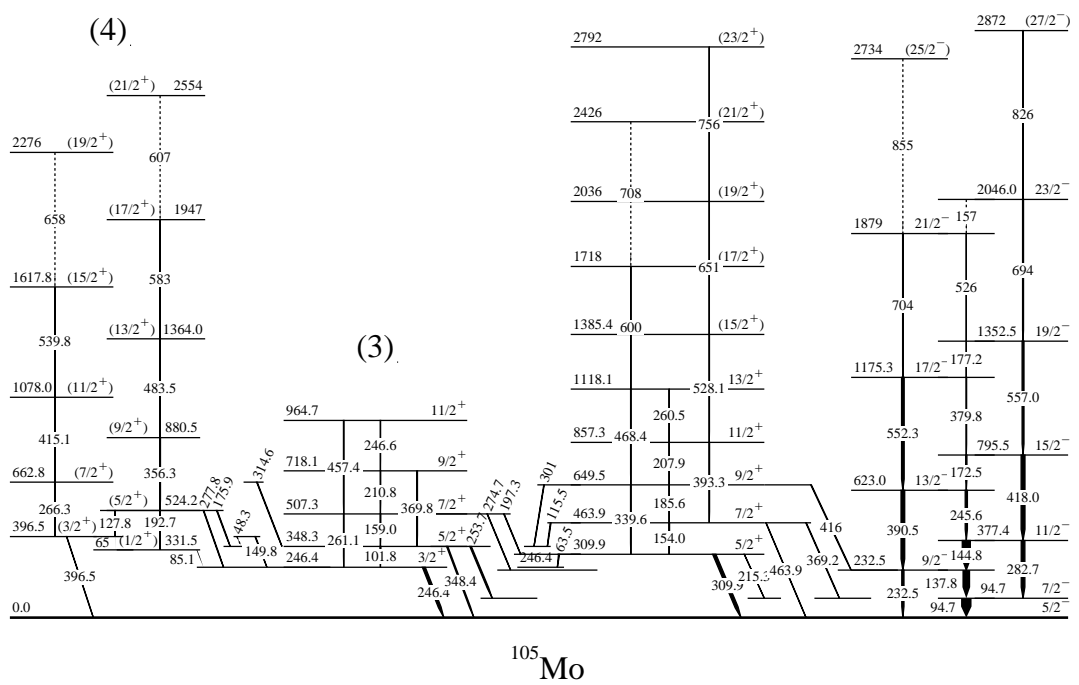

Fig. 5. Partial level scheme of ${ }^{105} \mathrm{Mo}$ as obtained in this work.

Three well developed bands were previously reported in ${ }^{107}$ Mo from $\gamma$ ray measurements of the spontaneous fission of ${ }^{248} \mathrm{Cm}$ [12]. To complete the level scheme, the Lohengrin mass spectrometer has been used to search for delayed $\gamma$-rays and conversion electrons in this nucleus using the setup described in Section 1.2. A new isomer with a $420 \mathrm{~ns}$ half life was observed in ${ }^{107} \mathrm{Mo}$. It was tentatively assigned to be a $1 / 2^{+}$state deexciting by a $65.4 \mathrm{keV} E 2$ transition to the $5 / 2^{+}$ground state. A $30 \mathrm{keV}, \mathrm{E} 1,3.85 \mu \mathrm{s}$ transition from the $3 / 2^{+}$level of the $1 / 2^{+}[431]$ intruder band to the ground state was observed in ${ }^{107} \mathrm{Tc}$.

\subsection{Discussion}

The excited states of the bands in ${ }^{105,107} \mathrm{Mo}$ and ${ }^{107} \mathrm{Tc}$ and their $\gamma$-ray decay patterns were calculated using the code ASYRMO. In the present 
calculation, values $\kappa=0.068$ and $\mu=0.35$ were taken for the strength parameters of the $\mathbf{l s}$ and $\mathbf{l}^{2}$ terms, for both $N=4$ and $N=5$ neutron shells. The other parameters entering in the level calculations are pairing introduced via a standard $B C S$ calculation, the deformation parameters $\epsilon_{2}$ and $\gamma$ of the single-particle potential in the intrinsic system and the inertia parameter $a$ of the bands. Agreement with theory can be improved by introducing an ad hoc "Coriolis attenuation" parameter, $\xi$. An effective value of $\xi=0.7$ is common in these calculations. Finally, the number of variable parameters was minimized by imposing that all bands of the same nucleus have the same parameter values.

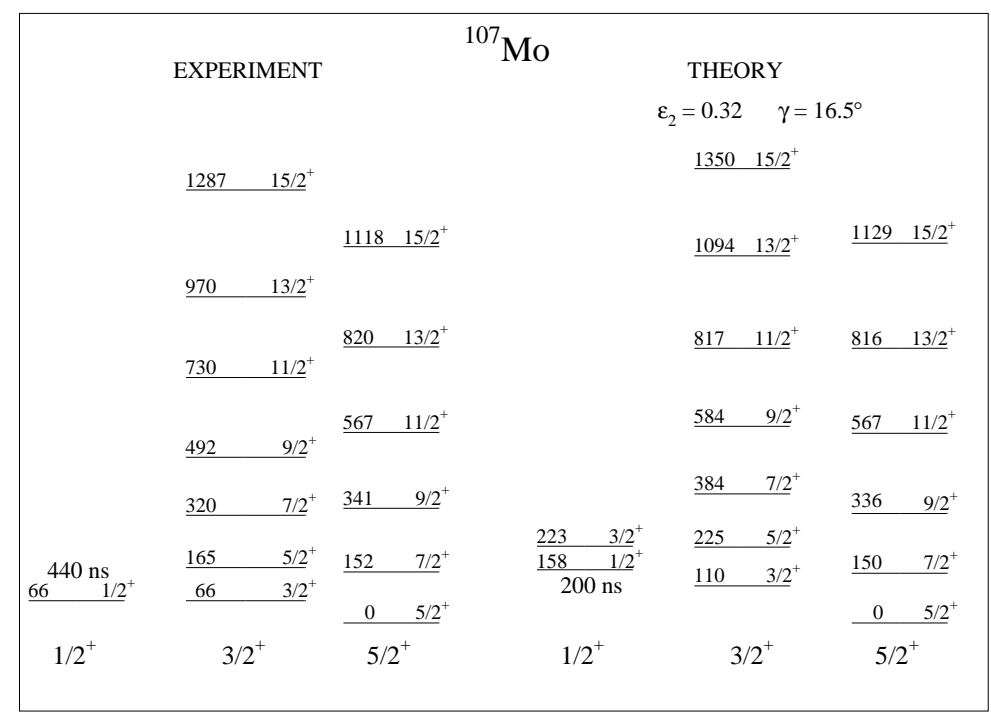

Fig. 6. Comparison of experimental and calculated positive-parity bands in ${ }^{107} \mathrm{Mo}$.

The calculations are able to correctly reproduce the four bands of the experimental level schemes only when deformations of $\epsilon_{2}=0.32$ and $\gamma \sim$ $17^{\circ}$ are used throughout. More details of the results of the calculation can be found in Ref. [13]. These values were compared to the deformations extracted for the neighboring even-even Mo nuclei, using the the Davydov and Filippov theory, and found to be very similar at low spins, suggesting that all these nuclei have similar core deformations. The isomeric state in ${ }^{107} \mathrm{Tc}$ and level scheme of ${ }^{105} \mathrm{Tc}$ have also been well reproduced using similar values in the calculations [14], showing that the odd proton does 
not greatly effect the deformation of the core.

\subsection{Summary}

New information on prompt $\gamma$-ray has been collected on ${ }^{105,107}$ Mo, comprising of four well-developed bands in ${ }^{105}$ Mo. New isomeric states have been reported for ${ }^{107} \mathrm{Mo}$ and ${ }^{107} \mathrm{Tc}$. These data have been successfully interpreted using simple quasiparticle-rotor model calculations, where deformations of $\epsilon_{2}=0.32$ and $\gamma \sim 17^{\circ}$ were found to correctly reproduce the experimental level schemes..

\section{References}

1. L. Coraggio, A. Covello, A. Gargano and N. Itaco, Phys. Rev. C 72, p. 057302 (2005).

2. L. Coraggio, A. Covello, A. Gargano and N. Itaco, Phys. Rev. C 73, p. 031302R (2006).

3. M. N. Mineva, M. Hellström et al., Eur. Phys. J. A 11, 9 (2001).

4. A. A. Sonzogni, Nuclear Data Sheets 95, p. 837 (2002).

5. G. S. Simpson, J. C. Angelique, J. Genevey, J. A. Pinston, A. Covello, A. Gargano, U. Köster, R. Orlandi and A. Scherillo, Physical Review C (Nuclear Physics) 76, p. 041303 (2007).

6. P. Hoff, J. P. Omtvedt, B. Fogelberg, H. Mach and M. Hellström, Phys. Rev. $C$ 56, 2865(Nov 1997).

7. A. Covello, L. Coraggio, A. Gargano and N. Itaco, Prog. Part. Nucl. Phys. 59, p. 401 (2007).

8. A. G. Smith, J. L. Durell, W. R. Phillips, M. A. Jones, M. Leddy, W. Urban, B. J. Varley, I. Ahmad, L. R. Morss, M. Bentaleb, A. Guessous, E. Lubkiewicz, N. Schulz and R. Wyss, Phys. Rev. Lett. 77, 1711(Aug 1996).

9. A. Guessous, N. Schulz, M. Bentaleb, E. Lubkiewicz, J. L. Durell, C. J. Pearson, W. R. Phillips, J. A. Shannon, W. Urban, B. J. Varley, I. Ahmad, C. J. Lister, L. R. Morss, K. L. Nash, C. W. Williams and S. Khazrouni, Phys. Rev. C 53, 1191(Mar 1996).

10. H. Hua, C. Y. Wu, D. Cline, A. B. Hayes, R. Teng, R. M. Clark, P. Fallon, A. Goergen, A. O. Macchiavelli and K. Vetter, Phys. Rev. C 69, p. 014317(Jan 2004).

11. J. Skalski, S. Mizutory and W. Nazarewicz, Nucl. Phys. A. 617, p. 282 (1997).

12. W. Urban, T. Rzaca-Urban, J. A. Pinston, J. L. Durell, W. R. Phillips, A. G. Smith, B. J. Varley, I. Ahmad and N. Schulz, Physical Review C (Nuclear Physics) 72, p. 027302 (2005).

13. J. A. Pinston, W. Urban, C. Droste, T. Rzaca-Urban, J. Genevey, G. Simpson, J. L. Durell, A. G. Smith, B. J. Varley and I. Ahmad, Physical Review C (Nuclear Physics) 74, p. 064304 (2006).

14. G. Simpson, J. Genevey, J. A. Pinston, U. Köster, R. Orlandi, A. Scherillo and I. A. Tsekhanovich, Physical Review C (Nuclear Physics) 75, p. 027301 (2007). 